\title{
Autorregulación afectivo- motivacional, resolución de problemas y rendimiento matemático en Educación Primaria
}

\section{Affective and motivational self-regulation, resolution of problems and performance in the area of mathematics in Primary Education}

\author{
Marta Martínez Vicente*1 \\ Mmv3619@hotmail.com \\ Carlos Valiente Barroso** \\ carlosvbsiete@hotmail.com \\ *Universidad Nacional de Educación a Distancia (UNED), España \\ **Instituto Clínico y de Investigación Interdisciplinar en Neurociencias, Santander, España
}

\section{Resumen:}

En los procesos de autorregulación del aprendizaje intervienen estrategias cognitivas y motivacionales fruto de las cuales los estudiantes generan creencias de autoeficacia, utilidad y valor de la tarea. Este estudio se propone analizar el efecto del componente afectivo-motivacional en resolución de problemas y el rendimiento matemático en general. Participaron 146 alumnos de primaria, a los que se les aplicó la prueba de resolución de problemas de la batería EVAMAT y un cuestionario ad hoc elaborado para evaluar cuatro dimensiones relacionadas con las actitudes hacia las Matemáticas. El análisis MANOVA informa de diferencias estadísticamente significativas en valoración del docente, utilidad y satisfacción, competencia

\begin{abstract}
:
In the processes of self-regulation which mediate learning cognitive and motivational strategies occur. They generate a belief in self-efficiency, usefulness and value of the task. The aim of this study is to analyze the effect of affective and motivational components in the resolution of problems and in mathematical performance in general. 146 Primary School pupils completed a test based on the resolution of problems contained in EVAMAT and in an ad hoc questionnaire elaborated in order to assess four dimensions related to attitudes towards mathematics. A MANOVA analysis revealed statistically significant differences in teacher assessment, usefulness and satisfaction, mathematical competence and beliefs in resolution of
\end{abstract}

1 Dirección para correspondencia (correspondence address):

Marta Martínez Vicente. C/ Hermandad de Donantes de Sangre, 1, 3 B. 39200 Reinosa (España). 
Autorregulación afectivo-motivacional, resolución de problemas y rendimiento matemático en Educación Primaria

Marta Martínez Vicente y Carlos Valiente Barroso

matemática y creencias en resolución de problemas en función del curso, descendiendo las puntuaciones a medida que los alumnos avanzan en su escolaridad. Además, los alumnos con rendimiento alto obtienen puntuaciones mayores en dichas variables, que son al tiempo predictoras del rendimiento matemático en general. Se concluye enfatizando la necesidad de profundizar en la dimensión emocional del aprendizaje matemático que puede revertir en una experiencia más significativa, útil y funcional, incrementando la implicación y el interés del alumnado y en consecuencia el rendimiento académico en dicha área curricular.

\section{Palabras clave:}

Aprendizaje autorregulado; componente afectivo-motivacional; resolución de problemas y rendimiento matemático. problems according to level, showing that the marks go down as the pupils grow up. Furthermore, higher proficiency pupils obtain better results for these variables, which predict mathematical performance in general. The conclusion emphasizes the need to insist on the emotional dimension of mathematical learning, which can lead to a more significant, useful and functional experience, while at the same time, increase the implication and interest of the pupils and, consequently, academic performance in this curricular area.

\section{Key words:}

Self-regulated learning; affective and motivational learning; resolution of problems and mathematical performance.

\section{Résumé :}

Des stratégies cognitives et motivationnelles grâce auxquelles les étudiants génèrent des sentiments d'auto-efficacité, d'utilité et de valeur de la tâche interviennent dans les processus d'autorégulation de l'apprentissage. Cette étude propose d'analyser l'effet du composant affecto-motivationnel dans la résolution de problèmes et dans le rendement mathématique en général. 146 élèves de l'école primaire y ont participé. Ils ont passé l'épreuve de résolution de problèmes de la série EVAMAT et un questionnaire ad hoc élaboré en vue d'évaluer 4 dimensions liées aux attitudes envers les mathématiques. L'analyse MANOVA rapporte des différences statistiquement significatives au niveau de I'évaluation de l'enseignant, I'utilité et la satisfaction, la compétence mathématique et les croyances dans la résolution de problèmes en fonction du cours, constatant que les notes baissent au fur et à mesure que les élèves avancent dans leur scolarité. De plus, les élèves qui ont un rendement élevé obtiennent de meilleures notes dans ces variables, qui prédisent en même temps le rendement mathématique en général. On conclut en insistant sur la nécessité d'approfondir la dimension émotionnelle de l'apprentissage mathématique qui peut aboutir à une expérience plus significative, utile et fonctionnelle, en augmentant ainsi l'implication et l'intérêt de l'élève et en conséquence le rendement académique dans la dite matière.

\section{Mots clés:}

Apprentissage auto-réglé; composant affecto-motivationnel; résolution de problèmes et rendement mathématique.

Fecha de recepción: 24-1-2018

Fecha de aceptación: 7-10-2018 


\section{Introducción}

Nuevas teorías cognitivas y constructivistas en psicología y educación centradas en la optimización del aprendizaje, ponen énfasis en los procesos de adquisición del conocimiento y no tanto en los resultados, atribuyendo una relevancia especial a las estrategias de aprendizaje de los alumnos, la función mediadora del docente, y los procesos conscientes que ambos emplean cuando se enfrentan a las tareas. Así, se abre un extenso espacio de investigación en el que se relacionan las variables cognitivas y motivacionales con el rendimiento académico, destacando sus correlaciones positivas con las estrategias de aprendizaje (Pintrich y Degroot, 1990). Hasta principios de los años 90 del pasado siglo, los aspectos motivacionales y cognitivos del aprendizaje escolar fueron estudiados independientemente. García y Pintrich (1994) lo constatan diferenciando los modelos motivacionales que explican el porqué del esfuerzo y la implicación en el trabajo escolar y los cognitivos que profundizan en los procesos de dominio y comprensión del mismo, utilizando diversas fuentes cognitivas y metacognitivas influenciadas notablemente por su implicación emocional. Núñez y González-Pumariega (1996) reclaman un aprendizaje en el que el alumno sea capaz de enfrentarse cognitivamente y motivado hacia las tareas, justificando así la integración en los modelos actuales de ambas dimensiones del proceso de aprendizaje.

Un nuevo paradigma de aprendizaje donde se integran los aspectos cognitivos, afectivo-motivacionales (Castillo, Balaguer y Duda, 2001; González-Pienda, Núñez, González-Pumariega y García, 1997; González-Pienda et al., 2000; Núñez et al., 1998; Suárez, González-Cabanach y Valle, 2001; Valle, González-Cabanach, Núñez, Rodríguez y Piñeiro, 2001) y conductuales (Boekaerts, Pintrich y Zeidner, 2000), siguiendo modelos que explican las relaciones entre motivación, metas académicas, emociones y volición con comportamientos que conllevan un aprendizaje efectivo (Boekaerts, 1999).

En el aprendizaje de las matemáticas se generan situaciones de ansiedad y estrés fruto de reacciones emocionales negativas intensas, que interfieren en el aprendizaje y aumentan según los alumnos van ascendiendo de curso escolar. La regulación emocional resulta esencial en la motivación hacia el aprendizaje, la autoestima y el éxito académico (Bisquerra, Pérez-González y García, 2015). El rendimiento escolar está 
influenciado por las emociones positivas que a su vez se relacionan con mayor creatividad, esfuerzo, implicación en las tareas, flexibilidad, toma de decisiones, pensamiento fluido y resolución de problemas (Pons, Hancock, Lafortune y Doudin, 2005). Por lo tanto, debe considerarse la dimensión emocional del aprendizaje para diseñar prácticas de enseñanza que aumenten la productividad y el interés de los estudiantes en su propio proceso de crecimiento académico y personal.

\section{Marco teórico}

El aprendizaje autorregulado se ha convertido en objeto de análisis y debate considerándole un constructo esencial en los procesos de aprendizaje (Rosário et al, 2010), dada su influencia directa con el rendimiento escolar. Diversas investigaciones revelan la importancia de variables motivacionales sobre la autorregulación del aprendizaje, consolidándose como tópicos cruciales del éxito escolar (Schunk y Zimmerman, 2008). Mediante el aprendizaje autorrregulado los estudiantes activan y conservan afectos y comportamientos junto con las cogniciones correspondientes (Rosário et al., 2009). Así se justifica que, a los primeros estudios sobre autorregulación del aprendizaje escolar centrados en el impacto de las estrategias cognitivas sobre éste (Zimmerman, 2008), se añadan las variables motivacionales demostrada su influencia sobre el proceso enseñanza-aprendizaje y el carácter predictivo sobre el rendimiento académico (Zimmerman, Bandura y Martínez-Pons, 1992). Estrategias reguladoras del esfuerzo y la motivación que pueden crear obstáculos para mantener un autoconcepto positivo, incitar una imagen negativa de sí mismo (pesimismo defensivo) o activar las dimensiones positivas del yo, eliminando las negativas, explicando la obtención de fracasos o éxitos al eliminar la ansiedad e incrementar el esfuerzo (García y Pintrich, 1994).

Revisada la literatura existente encontramos estudios que explican las relaciones directas y positivas del rendimiento académico con las metas de aprendizaje, las estrategias de comprensión y organización, así como con las estrategias de autoeficacia y autorregulación (Barca, 2009; Barca, Almeida, Porto, Peralbo, y Brenlla, 2012; Boekaerts y Corno, 2005; Rosário, Soares, Núñez, González-Pienda y Rubio, 2004; Rosário, Mourão, Núñez y Solano, 2008; Valle, Cabanach, Rodríguez, Núñez y 
González-Pienda, 2006; Valle et al., 2009; Zimmerman, 2008). Algunos estudios evidencian que las estrategias de autorregulación, autoeficacia académica y automotivación para el trabajo escolar disminuyen con la edad -especialmente en la etapa de Secundaria- en la que se observa un notable descenso de la motivación, argumentado por los cambios fisiológicos, biológicos y psicológicos a los que los estudiantes tienen que enfrentarse en este período y que interfieren notablemente en los aspectos académicos (Valle et al., 2008).

La autorregulación favorece el control de las emociones negativas y permite afrontar situaciones estresantes o desmotivantes (Boekaerts, 1999). Emociones académicas de los estudiantes, significativa y directamente, relacionadas con estrategias de aprendizaje implicadas en la autorregulación del aprendizaje, los recursos cognitivos, el éxito y el rendimiento académico y, al tiempo todas ellas con la motivación (Pekrun, Goetz, Tizt y Perry, 2002). Los estudiantes autorregulados poseen conciencia y regulación emocional que les permite aproximarse al logro de una meta, sin desviarse hacia estímulos distractores. Esta capacidad de autorregulación emocional se relaciona con menos afectaciones negativas y disminución de los síntomas depresivos, influyendo significativamente el contexto escolar (Garaigordobil, 2001). La competitividad dentro de las aulas incrementa los niveles de ansiedad y estrés en los estudiantes (Smith, Sinclair y Chapman, 2002), los docentes deben conseguir estrategias motivacionales instruccionales para el aprendizaje, a través de intervenciones basadas en el desarrollo de habilidades motivacionales autorreguladoras que incrementen la capacidad adaptativa de los alumnos en beneficio del éxito académico (Massone y González, 2007).

Dentro del componente afectivo-motivacional se incluyen las creencias sobre la capacidad, las metas y la eficacia de los estudiantes y queda constatado -a pesar de que los estudios empíricos no se han centrado en este tipo de estrategias motivacionales- en investigaciones recientes que proponen modelos para comprender cómo las estrategias de autorregulación motivacional relacionadas con el componente de valor (Suárez, Fernández, Rubio y Zamora, 2016), las expectativas, el valor y el afecto (Suárez y Anaya, 2005; Suárez y Fernández, 2011, 2013) inciden sobre las estrategias cognitivas y metacognitivas y por lo tanto en el rendimiento académico. Investigaciones fundamentadas en la utilización por parte de los estudiantes de estrategias de automotivación o motivacionales que 
provocan emociones positivas o negativas hacia el aprendizaje escolar (Boekaerts, 1996; García y Pintrich, 1994) influyendo notablemente en la persistencia y el esfuerzo en las tareas y consecuentemente en los resultados académicos (Mato, Espiñeira y Chao, 2014; Zimmerman, 2011).

El aprendizaje matemático, condicionado por múltiples factores, es el objetivo de algunas investigaciones realizadas durante los últimos años para dar respuesta al bajo rendimiento escolar en esta área curricular y los comportamientos de algunos estudiantes al enfrentarse ante tareas relacionadas con dicha competencia básica. Al deficiente uso de estrategias efectivas de planificación, atencionales o de concentración se suman estados de ansiedad provocados por emociones negativas resultado de actitudes, motivaciones y creencias en los procesos de cálculo o resolución de problemas, justificando que haya que profundizar en el origen de muchas de las tensiones generadas y los rechazos de los alumnos hacia esta asignatura.

El interés o aprecio en la resolución de problemas depende del componente afectivo-motivacional del aprendizaje. Durante la trayectoria escolar los estudiantes generan y acumulan ideas sobre sí mismos relacionadas con dicho proceso influyendo en su confianza, autoeficacia percibida y autoconcepto. Algunas de estas actitudes y comportamientos frente a la resolución son de pesimismo, frustración, rechazo o evitación (Guerrero, Blanco y Vicente, 2002), directamente relacionadas con las estrategias de afrontamiento que dispongan ante tales situaciones. El fracaso continuado provoca malestar, actitudes negativas y baja autoestima, justificando el abandono de la tarea ante la elevada ansiedad generada (Gómez-Chacón, 1997).

Con frecuencia se atribuye a las variables motivacionales y emocionales la explicación de por qué algunos alumnos fracasan al resolver problemas aun teniendo buenas capacidades cognitivas frente a otros que, con más constancia y perseverancia, mantienen el interés afrontando las dificultades que suponen para ellos este tipo de actividades. El modelo socioconstructivista contempla como las emociones -directamente relacionadas con el aprendizaje- se constituyen por procesos cognitivos, fisiológicos y motivacionales interrelacionados entre sí. Se ha estudiado la ansiedad, junto a las atribuciones, actitudes y creencias hacia las matemáticas, como elementos del componente afectivomotivacional (Tárraga, 2008; Zan, Brown, Evans y Hannula, 2006), a los que hay que añadir otro tipo de experiencias metacognitivas resultantes 
de los sentimientos generados en un alumno cuando se enfrenta a un problema matemático, la impresión de su dificultad y sus expectativas de resolución exitosa (Zan et al., 2006). Las competencias básicas de planificación o aplicación de estrategias de extracción de resultados, definidas en los currículos escolares, son inseparables de actitudes personales de perseverancia o interés en la tarea que facilitan el afrontamiento en resolución de problemas y el control emocional lo que repercute a su vez en un mayor rendimiento (Caballero, Guerrero, Blanco y Piedehierro, 2009), motivo por el cual hay que cuestionarse sobre cuál es el problema real en la enseñanza de las Matemáticas y buscar la solución desde el aprendizaje por competencias planteado legislativamente en la LOE y con continuidad en la LOMCE, priorizando los aprendizajes en contextos funcionales y prácticos (Goñi, 2009).

Se han llevado a cabo diversas investigaciones para explicar las percepciones de utilidad, satisfacción y confianza que tienen los estudiantes en el área de Matemáticas, siendo las variables género y curso las más incluidas (González-Pienda et al., 2012). Algunos de estos estudios reflejan que los chicos informan de mayor gusto por esta asignatura, les resulta más fácil y necesaria para su futuro profesional, se sienten más seguros y motivados (Frenzel, Pekrun y Goetz, 2007; Preckel, Goetz, Pekrun y Kleine, 2008) y muestran menos ansiedad que las chicas (ElseQuest, Hyde y Linn, 2010).

En cuanto al curso, estudios recientes informan del incremento de actitudes negativas hacia las Matemáticas según los estudiantes avanzan en su escolaridad. Con la edad disminuyen el interés, los sentimientos de utilidad y de significatividad, el autoconcepto, la confianza, la motivación y las creencias de autoeficacia (González-Pienda et al, 2006; Utsumi y Mendes, 2000; Watt, 2000; Warrington, Younger y Willians, 2000), aumentando significativamente la ansiedad hacia esta asignatura en particular.

En el campo de la Pedagogía y de la Psicología son escasos los estudios sobre educación matemática, fundamentales para trasladar los resultados a la práctica escolar y con ello mejorar la calidad del proceso de enseñanza-aprendizaje (Blanco, 2011). Es necesario realizar investigaciones empíricas que analicen las relaciones entre variables de corte motivacional y emocional en el proceso de enseñanza-aprendizaje de las matemáticas en general, y el de resolución de problemas en particular. Este trabajo se propone conocer las valoraciones que hacen los 
estudiantes de los docentes, la percepción de satisfacción, utilidad y competencia en el aprendizaje de las Matemáticas y las creencias y actitudes que tienen en la resolución de problemas matemáticos y así, poder identificar aquellos factores que interfieren en tal proceso.

\section{Método}

\section{Participantes}

Participaron en el estudio 146 estudiantes de Educación Primaria de un colegio concertado de la comunidad autónoma de Cantabria. Tipo de muestreo no probabilístico y casual. La muestra está compuesta por 63 niños $(43,2 \%)$ y 83 niñas $(56,8 \%)$, de edades comprendidas entre los 7 y los 13 años $(M=9.64, D T=1.65)$ correspondientes a los cursos $2 .^{\circ}, 3 .^{\circ}$, 4. ${ }^{\circ}, 5 .^{\circ}$ y $6 .^{\circ}$ de Educación Primaria.

\section{Instrumentos y variables}

Prueba EVAMAT para la Evaluación de la Competencia Matemática (García, García y González, 2011) de la que se recogieron los resultados de la prueba de resolución de problemas.

Para obtener información sobre las variables emocionales-motivacionales se elaboró un cuestionario ad hoc sobre creencias y actitudes hacia las Matemáticas tomando como referencia la revisión bibliográfica efectuada. Está compuesto por 24 ítems de escala tipo likert de 4 alternativas, desde 1 (nada) hasta 4 (mucho) referidos a cuatro dimensiones:

1. Valoración del docente, 8 ítems que se refieren a la percepción que tienen los alumnos sobre cómo enseña su profesor/a de Matemáticas.

2. Utilidad y satisfacción en el aprendizaje de las Matemáticas, 3 ítems que describen el agrado de los estudiantes hacia esta asignatura.

3. Competencia matemática, 4 ítems sobre el autoconcepto de los estudiantes en esta materia.

4. Creencias en resolución de problemas, 9 ítems sobre la percepción de los alumnos de su confianza y actitudes en el proceso de solución de problemas. 
Se analizó la fiabilidad del cuestionario a través de índice alfa de Cronbach obteniendo un valor de .876 para la escala total que informa de un buen nivel de consistencia interna.

Para medir el rendimiento académico en el área de Matemáticas, se recogió la calificación final del último trimestre del curso escolar.

\section{Procedimiento}

La aplicación de la prueba de resolución de problemas y del cuestionario elaborado se llevó a cabo en una sesión de clase dentro del horario escolar, previa autorización del equipo directivo y con la conformidad de los tutores. A los estudiantes se les explicó el objetivo del estudio garantizando la confidencialidad de los datos y su anonimato.

\section{Análisis de datos}

En este estudio no experimental, transversal, descriptivo y correlacional se utilizaron en el análisis técnicas estadísticas descriptivas y se realizaron análisis multivariados de la varianza (MANOVA) tomando el curso y el rendimiento matemático como factores y como variables dependientes la valoración docente, utilidad y satisfacción hacia las Matemáticas, competencia percibida y creencias en resolución de problemas. Se analizaron el contraste multivariado, el efecto principal de los dos factores y su interacción sobre cada una de las variables dependientes. El tamaño del efecto se midió con el coeficiente eta-cuadrado parcial $\left(\eta_{p}{ }^{2}\right)$, considerándolo pequeño cuando $\eta_{\mathrm{p}}{ }^{2}=.01(\mathrm{~d}=.20)$, medio cuando $\eta_{\mathrm{p}}{ }^{2}=.059$ $(\mathrm{d}=.50)$ y grande $\mathrm{si} \eta_{\mathrm{p}}^{2}=.138(\mathrm{~d}=.80)$ (Cohen, 1988). Para analizar las diferencias entre las variables vinculadas al componente afectivo-motivacional en función del rendimiento matemático se establecieron tres grupos según los percentiles: rendimiento bajo (puntuaciones inferiores al percentil 25), rendimiento medio (puntuaciones entre el percentil 25 y 75) y rendimiento alto (puntuaciones superiores al percentil 75).

Además se realizaron correlaciones de Pearson para observar las relaciones entre las variables y por último llevar a cabo un análisis de regresión múltiple que permitiese conocer el carácter predictivo de las variables mencionadas y el curso, sobre el rendimiento académico en Matemáticas.

El análisis de datos se realizó con el programa estadístico SPSS versión 24.0 para Windows. 


\section{Resultados y discusión}

\section{Análisis descriptivo y correlacional}

Primero se realizó un análisis descriptivo de los ítems para contextualizar cada una de las cuatro dimensiones analizadas en el cuestionario (tabla 1). En relación a la primera de ellas referida a la valoración del docente, destacan los porcentajes elevados en sus respuestas entre bastante y mucho, según los cuales les gusta como enseña $(48.5 \%)$, el/la profesor/a les aconseja (44.0\%) y se interesa en ayudarles (56\%) haciendo que les resulte fácil el aprendizaje de esta materia $(40.3 \%)$.

En cuanto a la utilidad y satisfacción hacia dicha asignatura, los porcentajes informan del gusto $(47 \%)$ y de la importancia que los estudiantes atribuyen a la misma (74.6\%). Asimismo se perciben competentes en el área de Matemáticas, aunque no consideran que sea la única materia que se les da bien (35.1\%).

Por último, los resultados en creencias en resolución de problemas matemáticos indican actitudes positivas de los estudiantes cuando se enfrentan a dichas tareas (confían en sí mismos entre bastante y mucho, $38.1 \%$ y $37.3 \%$ ) y con esfuerzo y paciencia es posible afrontar la resolución de los problemas (55.2\% y 67.2\%), proporcionándoles estados de ánimo positivos (58.2\%).

Tabla 1

Resultados de los ítems del cuestionario en porcentajes

\begin{tabular}{lcccc}
\hline Ítem & Nada & Poco & Bastante & Mucho \\
\hline Valoración del docente & & & & \\
\hline 1. Me gusta cómo enseña & 6.0 & 13.4 & 32.1 & 48.5 \\
2. Me aconseja y enseña & 4.5 & 13.4 & 38.1 & 44.0 \\
3. Me hace sentir que soy bueno & 7.5 & 18.7 & 44.8 & 29.1 \\
4. Se interesa por ayudarme & 3.7 & 13.4 & 26.9 & 56.0 \\
5. Se divierte cuando enseña & 8.2 & 21.6 & 41.0 & 29.1 \\
6. Le pregunto cuando no entiendo & 2.2 & 23.9 & 36.6 & 37.3 \\
7. Las clases son participativas & 12.7 & 16.4 & 35.1 & 35.8 \\
8. Hace que me resulten fáciles & 8.2 & 19.4 & 32.1 & 40.3 \\
\hline
\end{tabular}




\begin{tabular}{lcccc}
\hline Ítem & Nada & Poco & Bastante & Mucho \\
\hline Utilidad/Satisfacción & & & & \\
\hline 9. Me gustan & 6.7 & 13.4 & 32.8 & 47.0 \\
10. Nunca las dejaría & 7.5 & 16.4 & 34.3 & 41.8 \\
11. Son importantes & 4.5 & 2.2 & 18.7 & 74.6 \\
\hline Competencia matemática & & & & \\
\hline 12. Soy bueno & 3.7 & 15.7 & 55.2 & 25.4 \\
13. Son fáciles para mí & 5.2 & 21.6 & 40.3 & 32.8 \\
14. Se comprenden bien & 4.5 & 27.6 & 47.0 & 20.9 \\
15. Única materia que se me da bien & 34.3 & 35.1 & 18.7 & 11.9 \\
\hline Creencias en resolución de problemas & & & & \\
\hline 16. Me gusta resolver & 10.4 & 20.9 & 35.8 & 32.8 \\
17. Suelo dudar del resultado & 18.7 & 43.3 & 20.9 & 17.2 \\
18. Confío en mí mismo & 6.7 & 17.9 & 38.1 & 37.3 \\
19. Estoy calmado y tranquilo & 9.7 & 16.4 & 42.5 & 31.3 \\
20. Me doy por vencido enseguida & 38.1 & 34.3 & 13.4 & 14.2 \\
21. Resuelvo con más seguridad en grupo & 9.7 & 14.2 & 34.3 & 41.8 \\
22. Me pongo contento cuando resuelvo bien & 3.0 & 10.4 & 28.4 & 58.2 \\
23. La resolución exige esfuerzo y paciencia & 2.2 & 4.5 & 38.1 & 55.2 \\
24. Si me esfuerzo lo consigo & .7 & .7 & 31.3 & 67.2 \\
\hline
\end{tabular}

En la tabla 2 aparecen las correlaciones de Pearson entre las variables estudiadas, así como sus medias y desviaciones típicas. Considerando las correlaciones, el rendimiento matemático mantiene una relación estadísticamente significativa y positiva con el rendimiento en resolución de problemas $(r=.410 ; p<.01)$, con valoración del docente $(r=.352 ; p<.01)$, utilidad y satisfacción $(r=.339 ; \mathrm{p}<.01)$, competencia matemática $(r=.280$; $\mathrm{p}<.01)$ y creencias en resolución de problemas $(r=.222 ; \mathrm{p}<.05)$. Se evidencia la dependencia del rendimiento matemático con las actitudes de los estudiantes hacia dicha asignatura. La variable curso mantiene relaciones estadísticamente significativas inversas con el rendimiento matemático $(\mathrm{r}=-.173 ; \mathrm{p}<.05)$, resolución de problemas $(\mathrm{r}=-.205 ; \mathrm{p}<.05)$, valoración del docente $(r=-.354 ; \mathrm{p}<.01)$, utilidad y satisfacción hacia la materia $(\mathrm{r}=-.182 ; \mathrm{p}<.05)$, competencia matemática $(\mathrm{r}=-.217 ; \mathrm{p}<.05)$ y creencias en resolución de problemas $(\mathrm{r}=-.376 ; \mathrm{p}<.01)$, indicando que a medida que los alumnos ascienden de curso su rendimiento académico es menor y las creencias y motivaciones son más negativas que en los primeros cursos. 
En cuanto a las cuatro variables vinculadas al componente afectivoemocional, las relaciones son positivas y estadísticamente significativas entre valoración del docente y utilidad y satisfacción $(r=.543 ; \mathrm{p}<.01)$, competencia matemática $(\mathrm{r}=.509 ; \mathrm{p}<.01)$ y creencias en resolución de problemas $(\mathrm{r}=.591 ; \mathrm{p}<.01)$; asimismo entre utilidad y satisfacción y competencia matemática $(\mathrm{r}=.584 ; \mathrm{p}<.01)$ por un lado y creencias en resolución de problemas por otro $(\mathrm{r}=.571 ; \mathrm{p}<.01)$, variable esta última que también se relaciona significativa y positivamente con competencia matemática $(\mathrm{r}=.418 ; \mathrm{p}<.01)$.

Tabla 2

Matriz de correlaciones, medias y desviaciones típicas de las variables

\begin{tabular}{|c|c|c|c|c|c|c|c|c|}
\hline & 1 & 2 & 3 & 4 & 5 & 6 & 7 & 8 \\
\hline Rend.matemático & 1.000 & & & & & & & \\
\hline Rend.resolución & $.410^{* *}$ & 1.000 & & & & & & \\
\hline Edad & -.158 & $-.201 *$ & 1.000 & & & & & \\
\hline Curso & $-.173 *$ & $-.205^{*}$ & $.943^{* *}$ & 1.000 & & & & \\
\hline $\begin{array}{l}\text { Valoración do- } \\
\text { cente }\end{array}$ & $.352^{* *}$ & $.232 * *$ & $-.318^{* *}$ & $-.354^{* *}$ & 1.000 & & & \\
\hline $\begin{array}{l}\text { Utilidad/Satisfac- } \\
\text { ción }\end{array}$ & $.339 * *$ & $.227^{* *}$ & $-.192 *$ & $-.182^{*}$ & $.543^{* *}$ & 1.000 & & \\
\hline $\begin{array}{l}\text { Competencia } \\
\text { matemática }\end{array}$ & $.280^{* *}$ & $.237^{* *}$ & $-.210^{*}$ & $-.217^{*}$ & $.509 * *$ & $.584^{* *}$ & 1.000 & \\
\hline $\begin{array}{l}\text { Creencias resolu- } \\
\text { ción }\end{array}$ & $.222^{*}$ & $.211^{*}$ & $-.340^{* *}$ & $-.376^{* *}$ & $.591^{* *}$ & $.571^{* *}$ & $.418^{* *}$ & 1.000 \\
\hline$M$ & 7.13 & 60.33 & 9.63 & 3.12 & 3.09 & 3.31 & 2.74 & 2.99 \\
\hline DT & 2.01 & 28.59 & 1.71 & 1.47 & .64 & .64 & .61 & .41 \\
\hline
\end{tabular}

Para el análisis de la relación entre las cuatro variables estudiadas, valoración del docente, utilidad y satisfacción, competencia matemática y creencias en resolución de problemas matemáticos (variables dependientes) se llevó a cabo un análisis MANOVA con dos variables independientes (curso y rendimiento académico en Matemáticas). Los estadísticos descriptivos se muestran en la tabla 3. 
Tabla 3

Media y desviación típica de las variables "valoración docente", "utilidad/satisfacción", "competencia matemática" y "creencias en resolución de problemas matemáticos" en función del curso y del rendimiento matemático

\begin{tabular}{|c|c|c|c|c|c|c|c|c|}
\hline & \multicolumn{2}{|c|}{$\begin{array}{c}\text { Valoración } \\
\text { docente }\end{array}$} & \multicolumn{2}{|c|}{$\begin{array}{l}\text { Utilidad/ } \\
\text { satisfacción }\end{array}$} & \multicolumn{2}{|c|}{$\begin{array}{c}\text { Competencia } \\
\text { matemática }\end{array}$} & \multicolumn{2}{|c|}{$\begin{array}{c}\text { Creencias en } \\
\text { resolución }\end{array}$} \\
\hline & $M$ & DT & $M$ & DT & $M$ & DT & $M$ & DT \\
\hline \multicolumn{9}{|l|}{ 2. ${ }^{\circ}$ curso } \\
\hline R. bajo & 3.50 & . & 2.67 & . & 3.25 & . & 2.67 & . \\
\hline R. medio & 3.15 & .400 & 3.31 & .554 & 2.79 & .832 & 3.07 & .288 \\
\hline R. alto & 3.47 & .441 & 3.67 & .373 & 2.97 & .551 & 3.20 & .384 \\
\hline Total & 3.26 & .429 & 3.40 & .532 & 2.87 & .734 & 3.10 & .326 \\
\hline \multicolumn{9}{|l|}{ 3.er curso } \\
\hline R. bajo & 3.45 & .481 & 3.20 & .767 & 2.90 & .518 & 3.31 & .506 \\
\hline R. medio & 3.39 & .283 & 3.44 & .527 & 2.89 & .697 & 3.16 & .267 \\
\hline R. alto & 3.53 & .229 & 3.67 & .471 & 3.06 & .458 & 3.08 & .257 \\
\hline Total & 3.45 & .310 & 3.47 & .570 & 2.95 & .560 & 3.17 & .325 \\
\hline \multicolumn{9}{|l|}{$4^{\circ}$ curso } \\
\hline R. medio & 3.38 & .577 & 3.42 & .495 & 2.83 & .606 & 3.35 & .321 \\
\hline R. alto & 3.38 & .428 & 3.74 & .465 & 2.69 & .570 & 3.21 & .239 \\
\hline Total & 3.37 & .506 & 3.56 & .498 & 2.77 & .580 & 3.29 & .291 \\
\hline \multicolumn{9}{|l|}{$5 .^{\circ}$ curso } \\
\hline R. bajo & 2.10 & .992 & 2.17 & 1.049 & 2.04 & .67 & 2.31 & .799 \\
\hline R. medio & 2.82 & .773 & 3.33 & .521 & 2.75 & .527 & 2.99 & .291 \\
\hline R. alto & 3.16 & .640 & 3.14 & .716 & 2.75 & .500 & 2.79 & .197 \\
\hline Total & 2.76 & .820 & 3.07 & .798 & 2.62 & .602 & 2.82 & .476 \\
\hline \multicolumn{9}{|l|}{$6 .^{\circ}$ curso } \\
\hline R. bajo & 2.59 & .656 & 3.17 & 1.00 & 2.19 & .718 & 2.75 & .508 \\
\hline R. medio & 2.75 & .696 & 3.11 & .675 & 2.45 & .455 & 2.73 & .325 \\
\hline R. alto & 3.01 & .397 & 3.33 & .492 & 2.85 & .405 & 2.79 & .261 \\
\hline Total & 2.83 & .592 & 3.20 & .642 & 2.57 & .517 & 2.75 & .318 \\
\hline \multicolumn{9}{|l|}{ TOTALES } \\
\hline R. Вајо & 2.73 & .922 & 2.77 & .987 & 2.42 & .723 & 2.76 & .717 \\
\hline R. Medio & 3.05 & .627 & 3.31 & .556 & 2.73 & .637 & 3.04 & .353 \\
\hline R. Alto & 3.29 & .464 & 3.51 & .530 & 2.87 & .490 & 3.01 & .328 \\
\hline Total & 3.09 & .640 & 3.31 & .647 & 2.74 & .613 & 2.99 & .411 \\
\hline
\end{tabular}

Nota. R: Rendimiento 
Los resultados obtenidos a nivel multivariado indican que el efecto del curso $\left(\lambda_{\text {wilks }}=.633 ; \mathrm{F}_{(16,358)}=3.618 ; p<.001 ; \mathrm{\eta}_{\mathrm{p}}{ }^{2}=.108\right)$ y del rendimiento matemático $\left(\lambda_{\text {Wilks }}=.865 ; \mathrm{F}_{(8,234)}=2.204 ; p<.05 ; \eta_{\mathrm{p}}{ }^{2}=.070\right)$ son estadísticamente significativos, siendo el tamaño del efecto grande. Sin embargo la interacción entre curso y rendimiento matemático no es estadísticamente significativa $\left(\lambda_{\text {Wilks }}=.752 ; \mathrm{F}_{(28,423)}=1.242 ; p=.187 ; \mathrm{\eta}_{\mathrm{p}}{ }^{2}=.069\right)$.

Los análisis univariados de cada variable dependiente tomada por separado, indican diferencias estadísticamente significativas entre los cursos en valoración docente $\left(\mathrm{F}_{(4,120)}=7.579 ; p<.001 ; \eta_{\mathrm{p}}{ }^{2}=.202\right)$, utilidad/ satisfacción $\left(\mathrm{F}_{(4,120)}=3.054 ; p<.05 ; \eta_{\mathrm{p}}{ }^{2}=.092\right)$, competencia matemática $\left(\mathrm{F}_{(4,120)}=2.680 ; p<.05 ; \eta_{\mathrm{p}}{ }^{2}=.082\right)$ y creencias en resolución de problemas $\left(\mathrm{F}_{(4,120)}=10.280 ; p<.001 ; \mathrm{\eta}_{\mathrm{p}}{ }^{2}=.255\right)$, con un efecto del tamaño grande. Las puntuaciones son menores en todas las variables estudiadas según se asciende en el curso (ver figura 1).

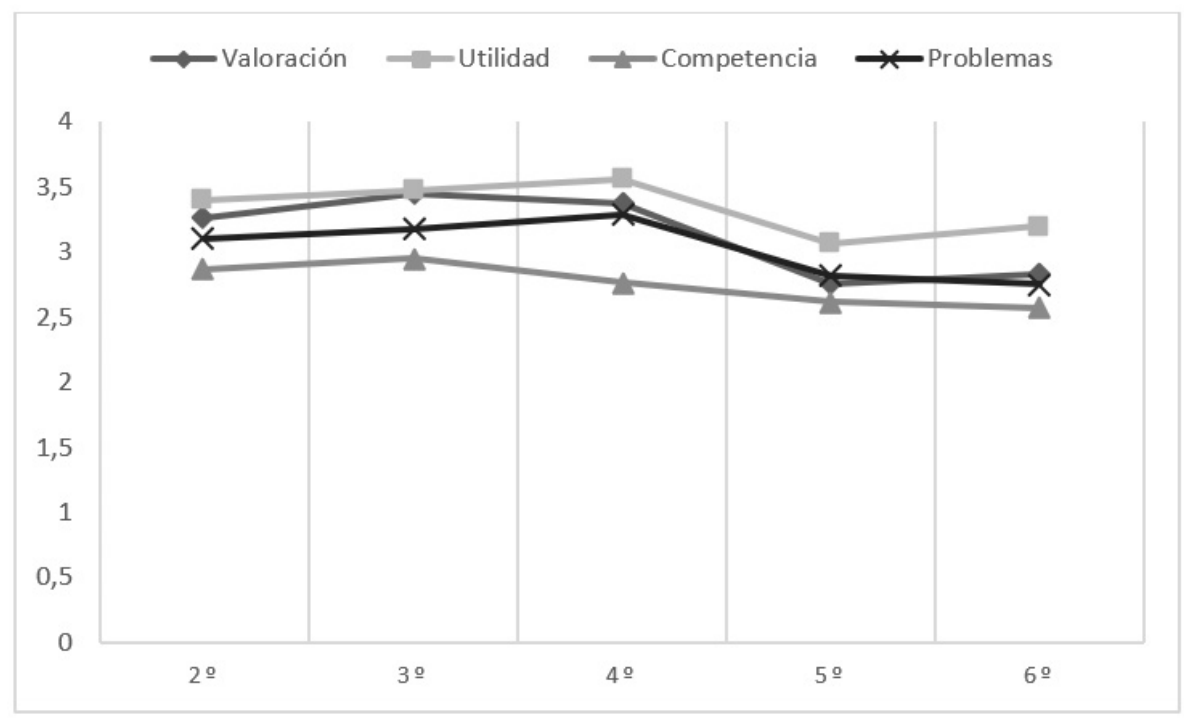

Figura 1. Representación gráfica de las variables vinculadas al componente afectivo-motivacional (valoración docente, utilidad/satisfacción, competencia y creencias en resolución) según el curso.

Los análisis univariados, en relación al rendimiento matemático, muestran que hay diferencias estadísticamente significativas en utilidad/satisfacción $\left(\mathrm{F}_{(2,120)}=5.182 ; p<.05 ; \eta_{\mathrm{p}}{ }^{2}=.080\right)$, con un efecto del tamaño grande, pero no hay diferencias estadísticamente significativas según el 
rendimiento académico en valoración docente $\left(\mathrm{F}_{(2,120)}=2.597 ; p=.079\right.$; $\left.\eta_{\mathrm{p}}{ }^{2}=.041\right)$, competencia matemática $\left(\mathrm{F}_{(2,120)}=1.111 ; p=.333 ; \eta_{\mathrm{p}}{ }^{2}=.018\right) \mathrm{y}$ creencias en resolución de problemas $\left(\mathrm{F}_{(2,120)}=2.076 ; p=.130 ; \eta_{p}{ }^{2}=.033\right)$.

Por último, indicar que la interacción curso y rendimiento matemático es estadísticamente significativa en creencias en resolución de problemas $\left(\mathrm{F}_{(7,120)}=2.430 ; p<.05 ; \eta_{\mathrm{p}}{ }^{2}=.124\right)$, efecto del tamaño grande, pero no ocurre lo mismo en valoración docente $\left(\mathrm{F}_{(7,120)}=1.092 ; p=.373 ; \eta_{\mathrm{p}}{ }^{2}=.060\right)$, utilidad/satisfacción $\left(\mathrm{F}_{(7,120)}=1.548 ; p=.158 ; \eta_{p}^{2}=.083\right)$, y competencia matemática $\left(\mathrm{F}_{(7,120)}=1.027 ; p=.416 ; \eta_{\mathrm{p}}^{2}=.057\right)$.

\section{Análisis de regresión}

Se realizó un análisis de regresión múltiple para estudiar el carácter predictivo de las cuatro variables vinculadas al componente afectivo-motivacional matemático sobre el rendimiento académico en esta misma materia. Se introdujeron dichas variables como predictoras para averiguar la cantidad de varianza y conocer en qué medida predicen el rendimiento matemático (tabla 4).

Tabla 4

Varianza explicada $\left(R^{2}\right)$, coeficientes de regresión $(\beta)$, estadístico t y significación asociada $\left(t_{(\mathrm{p}<)}\right)$ en la predicción del rendimiento matemático.

\begin{tabular}{lccc}
\hline & $\mathrm{R}^{2}$ & $\beta$ & $\mathrm{t}(\mathrm{p}<)$ \\
\hline RENDIMIENTO MATEMÁTICO & & & \\
\hline Primera etapa (Modelo 1) & .117 & & \\
Valoración docente & & .352 & $4.319^{* * *}$ \\
Segunda etapa (Modelo 2) & .142 & & \\
Valoración docente & & .238 & $2.487^{*}$ \\
Utilidad/Satisfacción & & .210 & $2.195^{*}$ \\
${ }^{* p<.05 * *}{ }^{*}<.01{ }^{* * *} p<.001$ & & &
\end{tabular}

En el modelo 1 (primera etapa) se observa que la variable valoración docente explica el $11.7 \%$ de la varianza total, siendo estadísticamente significativa $\left(\mathrm{F}_{(1,132)}=18.654 ; p<.001\right)$. Por tanto la variable valoración docente $(\beta=.352: t=4.319 ; \beta<.001)$ predice el rendimiento académico.

En el modelo 2 (segunda etapa) además de la variable valoración docente se introdujo la variable utilidad/satisfacción. Ambas variables explican conjuntamente el $14.2 \%$ de la varianza total, siendo estadísti- 
camente significativas $\left(\mathrm{F}_{(2,131)}=12.006 ; p<.001\right)$. Se concluye que, tanto valoración docente $(\beta=.238: \mathrm{t}=2.487 ; p<.05)$ como utilidad/satisfacción $(\beta=.210: t=2.195 ; p<.05)$ son variables relevantes en la explicación del rendimiento matemático. Las variables curso, competencia matemática y creencias de resolución de problemas fueron excluidas en ambos modelos.

\section{Conclusiones}

Los resultados obtenidos en este estudio informan de valoraciones positivas de los alumnos hacia su profesor/a de matemáticas, así como la relevancia que tiene para ellos esta materia académica, manifestada a través del gusto, satisfacción y utilidad de la misma y que posiblemente explique su autoconcepto matemático positivo. No obstante, no consideran que sea la única área curricular que se les da bien y en la que obtienen los mejores resultados. En relación a la resolución de problemas matemáticos afirman que es posible conseguirlo con paciencia y esfuerzo, apreciándose en general actitudes positivas hacia su aprendizaje. Las relaciones son significativas y positivas entre el rendimiento matemático y resolución de problemas matemáticos, con la valoración del docente, los sentimientos de utilidad y satisfacción hacia la materia, su competencia matemática, así como con las creencias que tienen cuando se enfrentan a tareas de resolución de problemas matemáticos. Como era de esperar atendiendo a investigaciones previas (González-Pienda et al., 2012; Mato et al., 2014), la variable curso muestra relaciones inversas con el rendimiento matemático, rendimiento en resolución de problemas y valoración docente por parte de los estudiantes. A medida que los alumnos cambian de curso su rendimiento es menor y tienen percepciones más negativas del docente.

Los alumnos con rendimiento alto en Matemáticas obtienen mayores puntuaciones en valoración docente, utilidad/satisfacción y creencias en resolución de problemas, mientras que los alumnos con rendimiento bajo se perciben con peor competencia matemática. Al mismo tiempo, el análisis de regresión constata que tanto la valoración que tienen los estudiantes de su docente, como la utilidad y satisfacción hacia esta materia de estudio, son predictoras del rendimiento matemático en general. Resultados en consonancia con otros anteriores que evidencian la rela- 
ción directa de las actitudes hacia las Matemáticas con el rendimiento académico en grupos heterogéneos de alumnos (Morales, 2006; Tárraga, 2008), siendo más negativas según progresan en la escolaridad obligatoria (González-Pienda, et al., 2006; Utsumi y Mendes, 2000; Watt, 2000). Este cambio de actitudes puede deberse a la falta de contextualización de lo aprendido, carente para ellos de aplicación funcional en la vida real (Mato et al., 2014). Por ello se proclama un aprendizaje con intencionalidad didáctica a partir del cual, el discente partiendo de la reflexión llegue a la internalización del propio proceso, consiguiendo un aprendizaje autónomo, estableciendo conexiones entre la realidad y las Matemáticas (Ortega, Pecharromán y Sosa, 2011).

La autorregulación emocional juega un papel esencial en la motivación hacia el aprendizaje y por lo tanto en el rendimiento resultado de dicho proceso. Los estudiantes deben evitar usar estrategias autorreguladoras motivacionales desadaptadas (Suárez et al., 2016), puesto que perder la autoconfianza en la capacidad y la competencia para aprender puede generar emociones negativas que disminuyan el interés, dando lugar a un menor esfuerzo e implicación en las tareas y por consiguiente a un menor rendimiento (Mato, 2010; Muñoz y Mato, 2008), emociones, actitudes y creencias que forman parte del componente afectivo (Blanco, 2008; Gómez-Chacón, 2000) y que predisponen al aprendizaje dando lugar a respuestas positivas o negativas que repercuten directamente en el rendimiento. Asimismo las percepciones que un estudiante tiene de su profesor, activan respuestas de aproximación o evitación hacia el aprendizaje de una materia y según sus experiencias previas se producirá un descenso o incremento del interés, de satisfacción y de conductas futuras hacia el aprendizaje en general (Bisquerra et al., 2015). Porque el profesor, guía todo un sistema de valores y creencias en los estudiantes (Murillo y Hernández, 2011) y debe conocer si los estudiantes disponen de una base sólida para fomentar actitudes positivas que les predisponga favorablemente al aprendizaje (Gil, Blanco y Guerrero, 2005), pudiendo así producir cambios significativos en su conducta ante el trabajo escolar (Fierro-Hernández, 2006).

Dentro de las limitaciones de esta investigación señalamos la necesidad de aumentar el tamaño de la muestra y el número de participantes de cada curso para cumplir adecuadamente los criterios de representatividad y obtener mayores garantías en la generalización de resultados. El hecho de que este estudio se haya realizado en un centro de una sola 
Autorregulación afectivo-motivacional, resolución de problemas y rendimiento matemático en Educación Primaria

Marta Martínez Vicente y Carlos Valiente Barroso

línea y de ratio reducida, en el que la enseñanza es más personalizada, no permite extraer conclusiones que reflejen la realidad del sistema educativo actual. Además, sería conveniente realizar estudios longitudinales incluyendo variables como titularidad de los centros (público o privado), género, edad y estilo de enseñanza del docente y las metas o atribuciones académicas de los estudiantes, para ofrecer resultados suficientemente sólidos y avalados científicamente. La mayor parte de los estudios se han realizado en Secundaria y en etapas superiores, por lo que profundizar en los primeros niveles de la escolaridad, cimiento de los aprendizajes posteriores, puede ser especialmente relevante en este tema.

A modo de síntesis, enfatizamos la necesidad de seguir realizando este tipo de estudios para ofrecer resultados que aporten funcionalidad y utilidad para toda la comunidad educativa. Una mayor investigación incluyendo la dimensión emocional en el aprendizaje matemático, puede revertir en un cambio de las percepciones y las actitudes hacia el mismo y consecuentemente en el rendimiento académico.

\section{Referencias}

Barca, A. (2009) (Coord.). Motivación y aprendizaje en contextos educativos. Granada: Grupo Editorial Universitario.

Barca, A., Almeida, L., Porto, A. M., Peralbo, M. y Brenlla, J. C. (2012). Motivación escolar y rendimiento: impacto de metas académicas, de estrategias de aprendizaje y autoeficacia. Anales de Psicología, 28(3), 848-859.

Blanco, L. J. (2008). Una revisión crítica de la investigación sobre las actitudes de los estudiantes universitarios hacia la Estadística. Revista Complutense de Educación, 19(2), 311-330.

Blanco, L. J. (2011). La Investigación en Educación Matemática. Educatio Siglo XXI, 29(1), 109-128.

Bisquerra, R., Pérez-González, J. C. y García, E. (2015). Inteligencia emocional en educación. Madrid: Síntesis.

Boekaerts, M. (1996). Self-regulated learning at the junction of cognition and motivation. European Psychologist, 2, 100-112.

Boekaerts, M. (1999). Self-regulated learning: Where are today. International Journal of Educational Research, 31, 445-458.

Boekaerts, M. y Corno, L. (2005). Self-Regulation in classroom: A perspective on assessment and intervention. Applied Psychology: An International Review, 54(2), 199231.

Boekaerts, M., Pintrich, P. R. y Zeidner, M. (2000). Handbook of self-regulation. San Diego: Academic Press. 
Autorregulación afectivo-motivacional, resolución de problemas y rendimiento matemático en Educación Primaria

Marta Martínez Vicente y Carlos Valiente Barroso

Caballero, A., Guerrero, E., Blanco, L. J. y Piedehierro, A. (2009). Resolución de problemas de matemáticas y control emocional. En M.J. González y J. MuriIlo (Eds.), Investigación en Educación Matemática XIII (pp. 151-160). Santander: SEIEM.

Cohen, J. (1988). Statistical power analysis for the behavioral sciences. Hillsdale, NJ: Erlbaum.

Castillo, I., Balaguer, I. y Duda, J. L. (2001). Perspectivas de meta de los adolescentes en el contexto académico. Psicothema, 13(1), 79-86.

Else-Quest, N. M., Hyde, J. S. y Linn, M. (2010). Cross-national patterns of gender differences in mathematics: A meta-analysis. Psychological Bulletin, 136(1), 103-127.

Fierro-Hernández, C. (2006). Valoración del impacto de un programa de educación en valores en el último curso de Educación Secundaria Obligatoria. Revista de Educación, 339, 455-466.

Frenzel, A. C., Pekrun, R. y Goetz, T. (2007). Girls and mathematics - A "hopeless" issue? A control-value approach to gender differences in emotions towards mathematics. European Journal of Psychology of Education, 22(4), 497-514.

Garaigordobil, M. (2001). Intervención con adolescentes: Impacto de un programa en la asertividad y en las estrategias cognitivas de afrontamiento en situaciones sociales. Psicología Conductual, 9(2), 221-246.

García Vidal, J., García Ortiz, B. y González Manjón, D. (2011). EVAMAT. Prueba para la evaluación de la competencia matemática. Madrid: EOS.

García, T. y Pintrich, P. R. (1994). Regulating motivation and cognition in the classroom: The role of self-schemas and self-regulatory strategies. En D.H. Schunk y B.J. Zimmerman, Self-regulation of learning and performance. Issues and educational applications. Hillsdale, NJ: LEA.

Gil, N., Blanco, L. y Guerrero, E. E. (2005). El papel de la afectividad en la resolución de problemas matemáticos. Revista de Educación, 340, 551-569.

Gómez-Chacón, I. M. (1997). La alfabetización emocional en educación matemática: actitudes, emociones y creencias. Revista Uno, 13, 7-22.

Gómez-Chacón, I. M. (2000). Matemática emocional. Los afectos en el aprendizaje matemático. Madrid: Narcea.

González-Pienda, J. A., Núñez, J.C. González-Pumariega, S. y García, M. (1997). Autoconcepto, autoestima y aprendizaje escolar. Psicothema, 9(2), 271-289.

González-Pienda, J. A., Núñez, J. C., González-Pumariega, S., Alvarez, L., Roces, C., García, M., González, P., Cabanach, R. y Valle, A. (2000). Autoconcepto, proceso de atribución causal y metas académicas en niños con y sin dificultades de aprendizaje. Psicothema, 12(4), 548-558.

Gonzalez-Pienda, J. A., Núñez, J. C., Solano, P., da Silva, E. H., Rosario, P., Mourâo, R. y Valle, A. (2006). Olhares de género face á matemática: uma investigaçao no ensino obrigatório español. Estudos de Psicología, 11(2), 135-141.

González-Pienda, J. A., Fernández-Cueli, M., García, T., Suárez, N., Fernández, E., Tuero-Herrero, E. y Helena da Silva, E. (2012). Diferencias de género en actitudes hacia las matemáticas en la enseñanza obligatoria. Revista Iberoamericana de Psicología y Salud, 3(1), 55-73. 
Autorregulación afectivo-motivacional, resolución de problemas y rendimiento matemático en Educación Primaria

Marta Martínez Vicente y Carlos Valiente Barroso

Goñi Zabala, J. M. (2009). El desarrollo de la competencia matemática en el currículo escolar de la Educación Básica. Educatio Siglo XXI, 27(1), 35-48.

Guerrero, E., Blanco, L. J. y Vicente, F. (2002). Trastornos emocionales ante la educación matemática. En J.N. García (Coord.), Aplicaciones para la intervención psicopedagógica. Madrid: Pirámide.

Massone, A. y González, G. (2007). Estrategias de afrontamiento y su relación con el logro académico en matemática y lengua en adolescentes de noveno año de educación general básica. Revista Iberoamericana de Educación.

Mato, M. D. (2010). Mejorar las actitudes hacia las matemáticas. Revista galego-portuguesa de psicoloxía e educación, 18(1), 1138-1663.

Mato, M. D., Espiñeira, E. y Chao, R. (2014). Dimensión afectiva hacia la matemática: resultados de un análisis en educación primaria. Revista de Investigación Educativa, 32(1), 57-72.

Morales, P. (2006). Medición de las actitudes en Psicología y Educación. Construcción de cuestionarios y problemas metodológicos. Madrid: Universidad Pontificia de Comillas.

Muñoz, J. M. y Mato, M. D. (2008). Análisis de las actitudes respecto a las matemáticas en alumnos de ESO. Revista de Investigación Educativa, 26(1), 209-226.

Murillo Torrecilla, F. J. y Hernández Castilla, R. (2011). Efectos escolares de factores socio-afectivos. Un estudio multinivel para Iberoamérica. Revista de Investigación Educativa, 29(2), 407-427.

Núñez, J. C., González-Pienda, J. A., García, M., González-Pumariega, S., Roces, C., Álvarez, L. y González-Torres, M. C. (1998). Estrategias de aprendizaje, autoconcepto y rendimiento académico. Psicothema, 10(1), 97-109.

Núñez, J. C. y González-Pumariega, S. (1996). Procesos motivacionales y aprendizaje. En J.A. González-Pienda, J. Escoriza, R. González y A. Barca, Psicología de la instrucción (2): Componentes cognitivos y afectivos del aprendizaje escolar. Barcelona: EUB.

Ortega, T., Pecharromán, C. y Sosa, P. (2011). La importancia de los enunciados de problemas matemáticos. Educatio Siglo XXI, 29(2), 99-116.

Pekrun, R., Goetz, T., Tizt, W. y Perry, R. P. (2002). Academic emotions in students'selfregulated learning and achievement: a program of qualitative and quantitative research. Educational Psychologist, 37(2), 91-105.

Pintrich, P. R. y Degroot, E. (1990). Motivational and self-regulated learning components of classroom acedmic perfomance. Journal of Educational Psychology, 82(1), 33-40.

Pons, F., Hancock, D. R., Lafortune, L. y Doudin, P-A. (Eds.). (2005). Emotions in Learning. Aalborg: Aalborg Universitetsforlag.

Preckel, F., Goetz, T., Pekrun, R. y Kleine, M. (2008). Gender differences in gifted and average-ability students: Comparing girls' and boys' achievement, self-concept, interest, and motivation in mathematics. Gifted Child Quarterly, 52, 146-59.

Rosário, P., Soares, S., Núñez, J. C., González-Pienda, J. y Rubio, M. (2004). Processos de auto-regulação da aprendizagem e realização escolar no ensino básico. Psicologia, Educação e Cultura, 8(1), 141-157.

Rosário, P., Mourão, R., Núñez, J. C. y Solano, P. (2008). Homework and Self-Regu- 
lated Learning (SRL) at issue: findings and future trends. En A. Valle., J.C. Núñez., R.G. Cabanach., J.A. González-Pienda y S. Rodríguez, Handbook of instructional resources and their applications in the classroom (pp. 123-134). New York: Nova Science.

Rosário, P., Mourão, M., Baldaque, M., Núñez, J. C., González-Pienda, J., Cerezo, R. y Valle, A. (2009). Tareas para casa, autorregulación del aprendizaje y rendimiento en matemáticas. Revista de Psicodidáctica, 14(2), 179-192.

Rosário, P., Núñez, J. C., González-Pienda, J., Valle, A., Trigo, L. y Guimarães, C. (2010). Enhancing self-regulation and approaches in first-year college students: A narrativebased program assessed in the Iberian Peninsula. European Journal of Psychology of Education, 25(4), 411-428.

Schunk, D. H. y Zimmerman, B. J. (2008) Motivation and Self-regulated learning. Theory, research and applications. New York: Lawrence Erlbaum.

Smith, L., Sinclair, K. E. y Chapman, E. S. (2002). Students' Goals, Self-Efficacy. Self-Handicapping, and Negative Affective Responses: An Australian Senior School Student Study. Contemporary Educational Psychology, 27, 471-485.

Suárez, J.M. y Anaya, D. (2005). Un modelo sobre la determinación motivacional del aprendizaje autorregulado. Revista de Educación, 338, 295-306.

Suárez, J. M. y Fernández, A. P. (2011). Evaluación de las estrategias de autorregulación afectivo-motivacional de los estudiantes: Las EEMA-VS. Anales de psicología, 27(2), 369-380.

Suárez, J. M. y Fernández, A. P. (2013). Un modelo sobre como las estrategias motivacionales relacionadas con el componente de afectividad inciden sobre las estrategias cognitivas y metacognitivas. Educación XXI, 16(2), 231-246. doi: 10.5944/educxx1.16.2.2641

Suárez, J. M., Fernández, A. P., Rubio, V. y Zamora, A. (2016). Incidencia de las estrategias motivacionales de valor sobre las estrategias cognitivas y metacognitivas en estudiantes de secundaria. Revista Complutense de Educación, 27(2), 421-435.

Suárez, J. M., González-Cabanach, R. y Valle, A. (2001). Multiple-goal pursuit and its relation to cognitive, self-regulatory, and motivational strategies. British Journal of Educational Psychology, 71, 561-572.

Tárraga, R. (2008). Relación entre rendimiento en solución de problemas y factores afectivo-motivacionales en alumnos con y sin dificultades de aprendizaje. Apuntes de Psicología, 26(1), 143-148.

Utsumi, M. C. y Mendes, C. R. (2000). Researching the attitudes towards mathematics in basic education. Educational Psychology, 20(2), 237-243.

Valle, A., González-Cabanach, R., Núñez, J. C. Rodríguez, S. y Piñeiro, I. (2001). Diferencias en la utilización de estrategias de aprendizaje según el nivel motivacional de los estudiantes. Revista de Investigación Educativa, 19, 105-126.

Valle, A., Cabanach, R., Rodríguez, S., Núñez, J. C. y González-Pienda, J. A. (2006). Metas académicas, estrategias cognitivas y estrategias de autorregulación del estudio. Psicothema, 18(2), 165-170.

Valle, A., Cabanach, R., González-Pienda, J. A., Núñez, J. C., Rodríguez, S. y Rosário, P. (2009). Perfiles motivacionales en estudiantes de secundaria: análisis diferencial 
Autorregulación afectivo-motivacional, resolución de problemas y rendimiento matemático en Educación Primaria

Marta Martínez Vicente y Carlos Valiente Barroso

en estrategias cognitivas, estrategias de autorregulación y rendimiento académico. Revista Mexicana de Psicología, 26(1), 113-124.

Valle, A., Núñez, J. C., Cabanach, R., González-Pienda, J. A., Rodríguez, S., Rosário, P., Cerezo, R. y Muñoz-Cadavid, M. (2008). Self-regulated profiles and academic achievement. Psicothema, 20(4), 724-731.

Warrington, M., Younger, M. y Williams, J. (2000). Student attitudes, image and the gender gap. British Educational Research Journal, 26(3), 393-407.

Watt, H. M. G. (2000). Measuring attitudinal change in mathematics and English over first year of junior high school: A multidimensional analysis. The Journal of Experimental Education, 68(4), 331-361.

Zan, R., Brown, L., Evans, J. y Hannula, M. S. (2006). Affect in mathematics education: an introduction. Educational Studies in Mathematics, 63, 113-121.

Zimmerman, B. J. (2008). Investigating self-regulation and motivation: historical background, methodological developments, and future prospects. American Educational Research Journal, 45, 166-183.

Zimmerman, B. J. (2011). Motivational sources and outcomes of Self-Regulated Learning and Performance. En B. J. Zimmerman y D. H. Schunk (Eds.), Handbook of SelfRegulation of Learning and Performance (pp. 49-64). New York: Rouledge.

Zimmerman, B. J., Bandura, A. y Martínez-Pons, M. (1992). Self-motivation for academic attainment: The role of self-efficacy beliefs and personal goal setting. American Educational Research Journal, 29, 663-676. 